\title{
Prediction of instantaneous heart rate using adaptive algorithms
}

\section{Sarita Kansal ${ }^{*}$}

Department of Electronics and Communication,

MITM/SOE,

Indore, M.P., India

Email: sarita_1411@yahoo.com

*Corresponding author

\section{Prashant P. Bansod}

Department Biomedical Engineering,

SGSITS,

Indore, M.P., India

Email: ppbansod43@gmail.com

\section{Abhay Kumar}

SoE,

D.A.V.V., Indore, M.P., India

Email: dr.abhaykumar@gmail.com

\begin{abstract}
In this paper, adaptive filter based on adaptive algorithms like least mean square (LMS), normalised least mean square (NLMS) and recursive least square (RLS) are used for the prediction of instantaneous heart rate in ECG signal. The adaptive algorithms work on the principle of optimising the least square error by achieving wiener solution. The weights of the filter coefficients are changing, as per the changes in the signal. The performance of adaptive filter is measured by mean square error (MSE) and the prediction accuracy is observed by mean absolute error (MAE). The simulation results show that the adaptive algorithms NLMS and RLS have faster convergence rate with less number of iteration but the forecasting accuracy is higher in LMS compared to NLMS and RLS algorithms.
\end{abstract}

Keywords: ECG; instantaneous heart rate; IHR; adaptive algorithm; least mean square; LMS; normalised least mean square; NLMS; recursive least square; RLS.

Reference to this paper should be made as follows: Kansal, S., Bansod, P.P. and Kumar, A. (2019) 'Prediction of instantaneous heart rate using adaptive algorithms', Int. J. Adaptive and Innovative Systems, Vol. 2, No. 4, pp.267-281.

Biographical notes: Sarita Kansal is a $\mathrm{PhD}$ scholar in Biomedical Signal Processing and Forecasting at School of Electronics, Devi Ahilya University, Indore, Madhya Pradesh, India. She received her Bachelor of Engineering in Electronics and Telecommunication Engineering from Baba Saheb Ambedkar University, Aurangabad, Maharastra, India and Master of Engineering in 
Telecommunication Technologies from Shri Govindram Sakseria Institute of Technology and Science, Indore, Madhya Pradesh, India. She is working as an Associate Professor at Medi-Caps Institute of Technology and Management, Indore. She has a master's dissertation with focus on networking and signal processing.

Prashant P. Bansod received his Bachelor of Engineering in Electronics and Instrumentation Engineering from Shri Govindram Sakseria Institute of Technology and Science, Indore, Madhya Pradesh, India, in July 1989 and the Master of Technology in Electronics Engineering from Visvesvariya Regional College of Engineering (now VNIT), Nagpur, India, in April 1992 and PhD in Biosciences and Bioengineering in 2010 from IIT Bombay. He worked as an Assistant Professor at S.G.S. Institute of Technology and Science from April 1993. He is currently working as a Professor and Head in Electronics and Instrumentation Engineering Department at SGSITS, Indore and also heading Biomedical Engineering Department. His fields of specialisation include medical signal and image processing, measurement systems and VLSI design. $\mathrm{He}$ persuaded research in field of medical signal and image processing and integrated sensors. He has published more than 30 research papers.

Abhay Kumar received his MSc in Physics and MTech in Opto-electronics and Optical Communication from Indian Institute of Technology, Delhi in 1987 and 1989 respectively. He received his $\mathrm{PhD}$ in Electronics from Devi Ahilya University, Indore in 2001. He started his professional career as a Lecturer at School of Electronics at Devi Ahilya University, Indore in 1990. Currently, he is a Professor and Chair, School of Electronics, Devi Ahilya University, Indore. He also served as Dean of Engineering Sciences during 2013-2015. His current research interests include application of signal processing algorithms such as compressive sensing for various biomedical applications. He has published more than 40 technical papers and has also served as general chairs/program chairs at various international symposia and conferences in his field.

\section{Introduction}

Adaptive filter technique uses different adaptive algorithms. It is used in many applications like noise cancellation, channel equalisation, system identification, echo cancellation, antenna arrays, line enhancement, controls and many more. It is also very popular and effective approach for processing and analysis of biomedical signals. One of the applications of adaptive filtering is to estimate the signal, by changing the values of the filter coefficients. The filter coefficients are changes in such a way that to map the input signal with the desired signal. It can be applied for the estimation of signals in non stationary environment (Shams et al., 2012).

In the research area of adaptive filtering very popular adaptive algorithms are least mean square (LMS), normalised least mean square (NLMS) and recursive least square (RLS). These algorithms may be applied for time series analysis and prediction. Researchers have used to forecast the variables in different fields like, LMS algorithm has been used in the area of finance for prediction of stock exchange prices (Wesen, 2015), and estimation of channels in wireless communication (Zia, 2009). It has been applied for the prediction of data in Wireless Sensor Network (Stojkoska et al., 2011). In the field of biomedical signals, LMS and NLMS algorithms have been used for the 
prediction of EOG signal (Naga Rajesh et al., 2012). The adaptive estimation algorithms with adaptive filters have also been used to predict the motion of the heart for robotically assisted beating heart surgery (Tuna et al., 2013) and non linear fast adaptive approach has been applied in paper (Liang et al., 2013) for the same. The adaptive algorithm RLS has been applied to detect the dynamic heart rate during intense physical activities using photoplethysmographic signals (Song et al., 2017). The adaptive algorithms are like an univariate time series model, where the prediction depends only upon the previous values of same variable, which need to be predicting for future.

The electrocardiogram (ECG) is an electrical signal which represents the direction and magnitude of electrical activity of human heart; it is generated by depolarisation and repolarisation of the atria and ventricles. The instantaneous heart rate (IHR) of an ECG signal is an important bio marker, which is used to diagnose the diseases related to heart rate like tachycardia, bradycardia etc. If the heart rate is predicted well is advanced, then it will be helpful for avoiding the cardiovascular diseases related to heart rate. In this paper, the prediction of IHR is done using adaptive algorithms. The performance of adaptive algorithms are evaluated by mean square error (MSE) with varying the parameters of adaptive filter and those are like the step size, filter length, number of iteration etc. and the prediction accuracy is measured by mean absolute error (MAE).

This paper is organised as follows: the introduction of adaptive filter and its uses for prediction is discussed in Section 2. Section 3 explained the error optimisation in adaptive filter using adaptive algorithm. Section 4 defined the bio marker 'IHR' of ECG. In the next Section 5, the simulation results and graphs are presented with analysis. The final Section 6 is concluded the paper based on the results and observations.

\section{Adaptive filter}

Adaptive filters are associated with adaptive algorithms. The adaptive algorithms continuously seek for the optimum value of error, using an orderly search process. It gives the superior performance compared to a system of fixed design (Windrow and Stearns, 2007). Theoretically the error optimisation is achieved by wiener solution but practically it is not possible. In wiener solution the filter coefficients becomes independent of time and solving for each instance of time would be impractical in most real time implementation. Therefore the iterative approach is used like steepest descent method (Haykin, 2003). The adaptive filter changes the filter coefficients iteratively to best map the input signal to the target signal. The difference between the target signal and filter output signal is known as error. This error is also called the cost function of adaptive filter. It is always desired to minimise the cost function of the adaptive filter (Moschytz and Hofbauer, 1992), which is between the desired and filter output signal. The cost function is commonly represented either by MSE or least square error (LSE).

The block diagram of an adaptive filter used for prediction is shown in Figure 1, where $d[n]$ is the targeted or desired signal, $x[n-k]$ is the delayed version of signal $x[n]$ at time instant $n$. The filtered output $y[n]$ at instant $n$ and the error $e[n]$ is the difference between $d[n]$ and $y[n]$. The coefficients or weights of an adaptive filter are adjusted in such a way to minimise the error. It is always desired to minimise the error (cost function) by following the output of the filter to the desired signal. The original signal $x[n]$ is applied to the input of the filter and initially the value of filter coefficients are zero. It is desired to adjust the filter coefficients in such a way that the output of the filter 
start following the input $x[n]$. That is why the desired signal $d[n]$ is connected to the input signal $x[n]$.

Figure 1 Block diagram of an adaptive filter for prediction

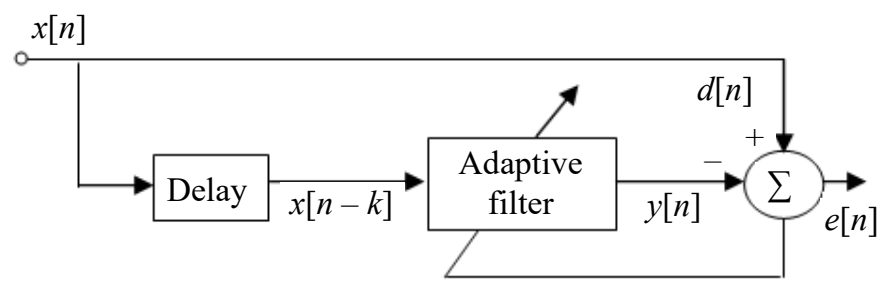

\section{Adaptive algorithm}

In this section, a brief discussion of adaptive algorithms LMS, NLMS and RLS is presented. LMS is invented by Widrow and Hoff (1960). It is based on the gradient estimation method, which is the improved steepest descent method. This algorithm is simple and numerically robust. It updates the weights of the filter at each time instant to minimise error (Kwong and Johnston, 1992) and it depends upon previous weight of the filter, step size, error and input signal $x[n]$. The LMS algorithm works as follows:

- $\quad$ initialise the filter length: $-M$ and step size: $-\mu$

- if the prior knowledge of tap weight vector $w[n]$ is not known or available, initialise it with $w[0]=0$

- the input vector at time instant $\mathrm{n}$ is $x[n]=[x[n], x[n-1], \ldots, x[n-M+1]]^{T}$ and $d[n]$ is the desired signal

- the output of the filter is $y[n]=w[n]^{T} \cdot x[n]$

- the estimated error is calculated as, $e[n]=d[n]-y[n]$

- the weight adaptation is $w[n+1]=w[n]+\mu x[n] e[n]$.

The last three steps are repeated to minimise the error and adapt the weights of the filter, till it gets converge.

In LMS algorithm the adjustment in weight vector of filter is directly proportional to the tap input vector $x[n]$. This leads to the problem of gradient noise amplification (Widrow and Stearns, 1985), when $x[n]$ is very large. This limitation of LMS is overcome by NLMS algorithm. The adjustment weight vector of NLMS is normalised with respect to the squared Euclidean norm of tap input vector $x[n]$. All the steps of NLMS algorithm is same as LMS algorithm, except the weight adaptation process as it is mentioned in equation (1), where $\|x[n]\|^{2}$ represents the squared Euclidean norm.

$$
w[n+1]=w[n]+\frac{\mu x[n] \cdot e[n]}{\|x[n]\|^{2}}
$$


RLS algorithm gives high performance and fast speed of convergence, when running in the time variant environment. The set of values or observation window is the time period under analysis. Which is usually has a rectangular or exponential fit. The parameter that controls the fit and duration of observation window is called the forgetting factor ' $\lambda$ '. It is related to the memory of the algorithm. The value of ' $\lambda$ ' is ranging from ' 0 ' to ' 1 ' and the objective function is given in equation (2)

$$
F(e(k))=\varepsilon^{d}(k)=\sum_{i=0}^{k} \lambda^{k-i} e^{2}(k)=\sum_{i=0}^{k} \lambda^{k-i}\left[d(i)-x^{T}(i) w(k)\right]^{2}
$$

This function is convex optimisation in multidimensional space $w(k)$ i.e., $\varepsilon^{d}(k)$ has one global minimum and no local minimum exist (Hayes, 1996). The error in RLS algorithm depends upon the forgetting factor ' $\lambda$ '. It gives the present error by considering the average of previous error.

\section{ECG marker IHR}

ECG signal gives vital information about the working of human heart. This vital information is indicated by the features of an ECG, which plays an important role for ECG diagnosis. The features are defined as Rpeak, Ppeak, Tpeak in terms of amplitude of the ECG and temporal intervals are like RRinterval, QTinterval, PRinterval etc. The value heart rate, beat rate and IHR are also used for diagnosis. The detail interpretation of these features and the method applied for detection of these features are explained in Kansal et al. (2015). The discrete wavelet transform is applied for feature extraction and then statistical parameters (mean and standard deviation) are used to obtain the markers. They are named as bio markers.

The ECG signal is represented by number of beats and each beat has the information of markers. The IHR is one of the signatures of ECG and extracted with the help of RRinterval. The term ' $R R$ ' indicates the representation of two consecutive Rpeaks and the time duration between them is called RRinterval. The details are available in Kansal et al. (2015). In every beat the value of IHR is calculated and at time instant ' $t$ ', the previous values of IHR represents the time series sequence or signal. The prediction of IHR means that if the previous values are known then the future values can be known by using adaptive algorithms.

\section{Simulation result and analysis}

The ECG records are collected from MIT-BIH ECG database (http://www.physionet.org/ physiobank/database/nsrdb/) for simulation purpose. The healthy ECG records of ten people are considered and studied the performance of adaptive algorithms for prediction. The ECG signals are resample at $360 \mathrm{~Hz}$ of frequency. The duration of five minute is considered from each record. 
Figure 2 Time series signal for IHR (see online version for colours)

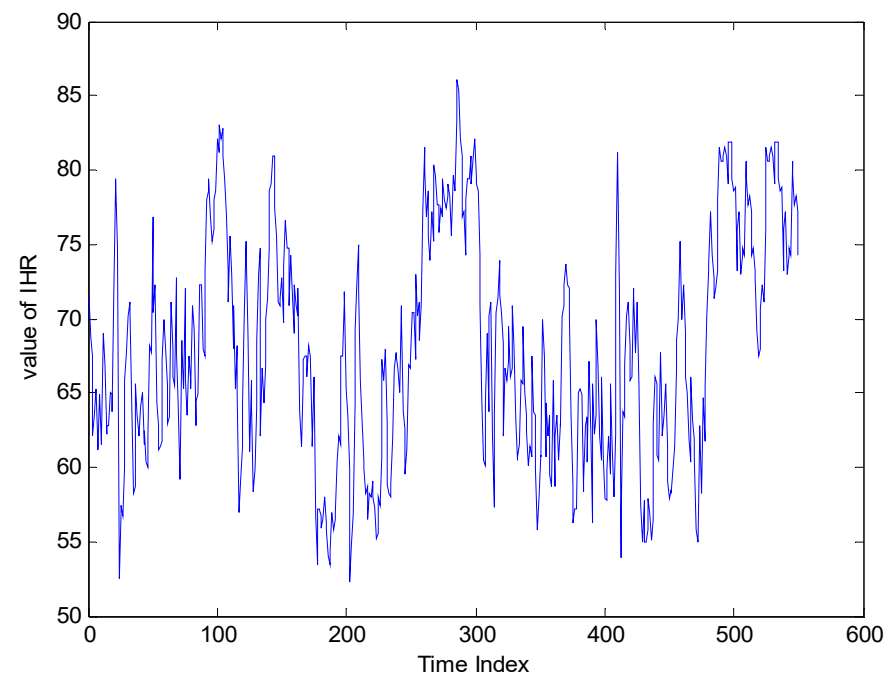

Figure 3 Normalised signal for IHR (see online version for colours)

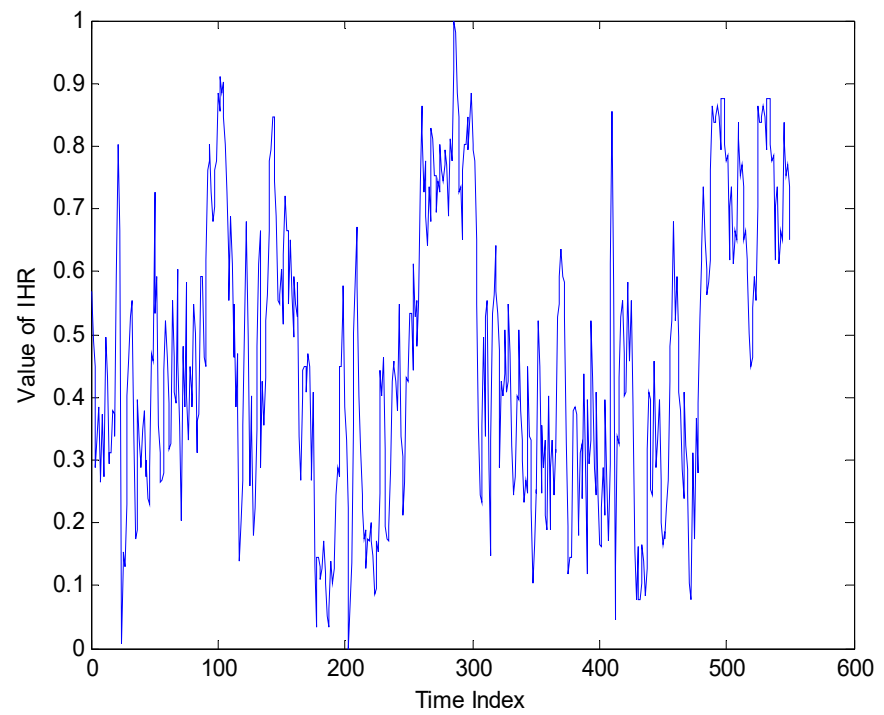

The time series signal of IHR is shown in Figure 2. The value of IHR is varying from 53 to 86 beats per minute. The normalised IHR signal is shown in Figure 3. This normalised signal is applied to evaluate the performance of adaptive algorithms for prediction. The total 550 samples of IHR are collected from one record of ECG and provided the delay of 50 because the length of forecasting horizon is assumed to be 50 . Therefore, the 500 samples are used for training and 50 samples are used for testing the system. 
In the first part of simulation setup, LMS algorithm is used in adaptive filter for prediction. The length of filter is kept constant, which is directly related to the past values of the signal used for prediction. The assumed values of step sizes are $0.001,0.005$, $0.009,0.01$ and 0.05 . The performance of LMS algorithm is evaluated with different step size and depicted in Figure 4. It is clear from Figure 4; the MSE is minimal at step size of 0.05 . The number of iteration required for convergence is also varying with step size.

Figure 4 LMS filter with different step size values (see online version for colours)

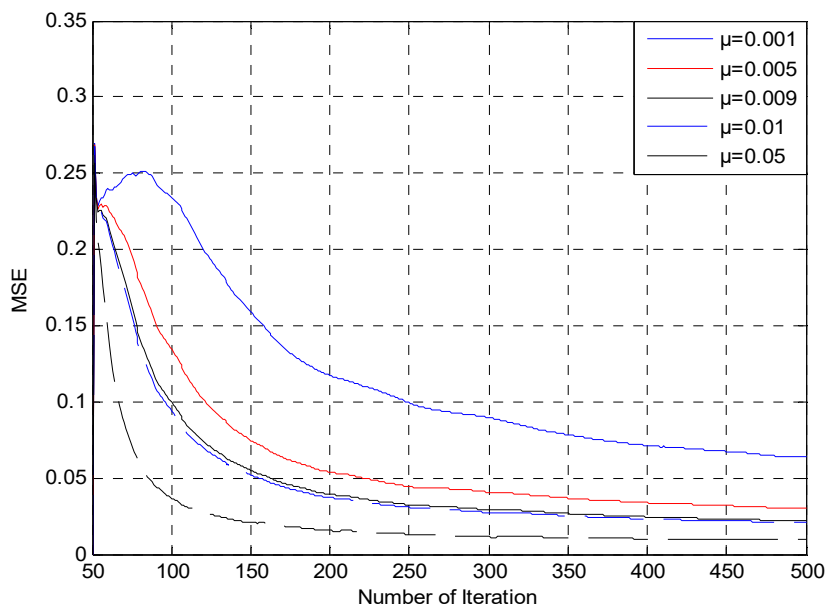

Figure 5 LMS filter with different step size values (see online version for colours)

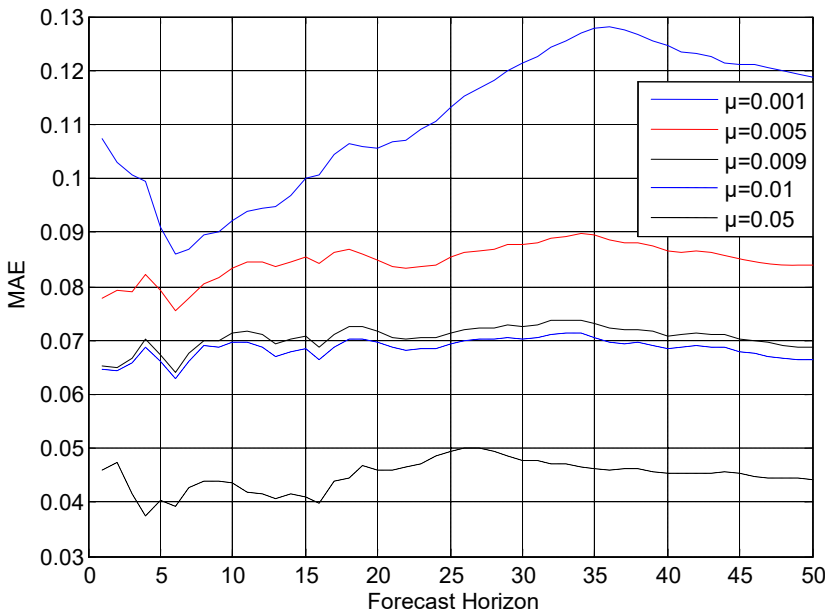

The filter is not performed well with step size higher than 0.05 , as the output is not following the desired signal. The prediction accuracy with different step size is shown in Figure 5 and the accuracy is higher at 0.05 . Therefore the step size 0.05 is considered for the next simulation setup. 
Figure 6 LMS filter with different filter length values (see online version for colours)

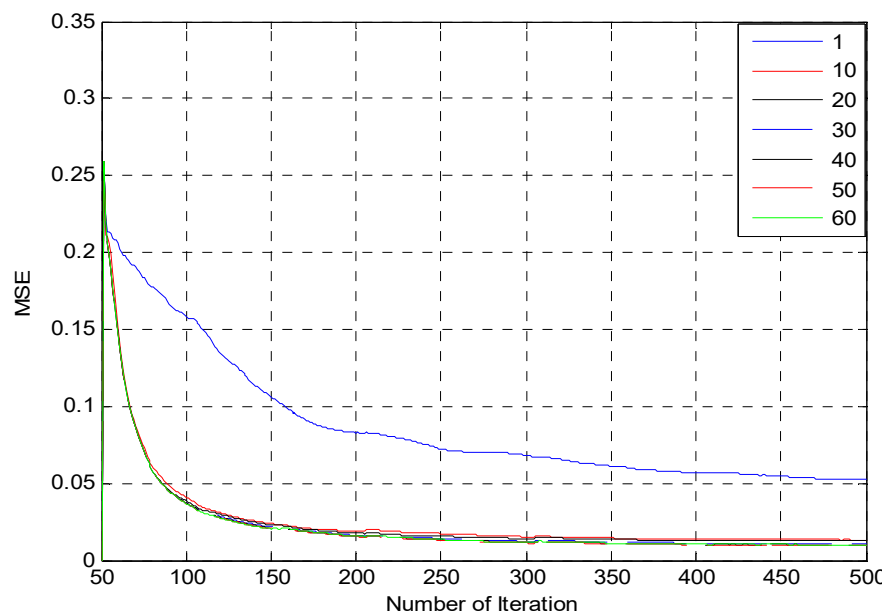

Figure 7 LMS filter with different filter length values (see online version for colours)

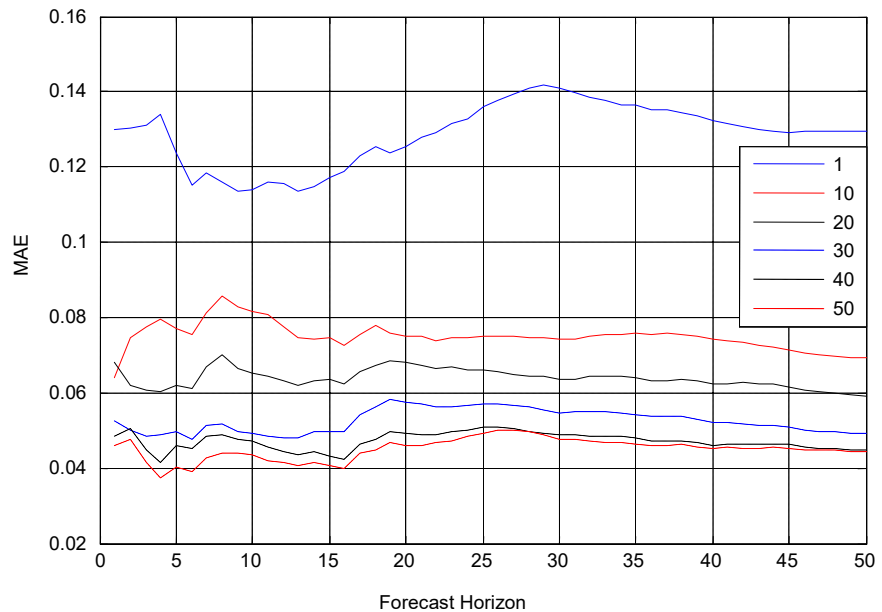

The filter length is increased from 1 to 100 with fixed step size 0.05 . In the given dataset the LMS algorithm worked up to the filter length 60. The performance of filter is affected by varying the length of the filter as shown in Figure 6, with number of iterations. It is evaluated that, as filter length is increasing the performance of filter is better up to some value. The increase in filter length includes more past values of the signal. The difference in performance of filter with filter length 1 and 10 can be easily observed by Figure 6 compare to other filter length. Therefore the performance of filter length 10 to 60 is more easily analysed by Figure 7. It depicts from Figure 7, that the filter length 50 is performed better than any other filter length by minimising MSE. Therefore, the step size 0.05 and filter length 50 can be used for LMS algorithm to predict the value of IHR. The results are presented by averaging the individual results of ECG records. 
Figure 8 NLMS filter with different step size values (see online version for colours)

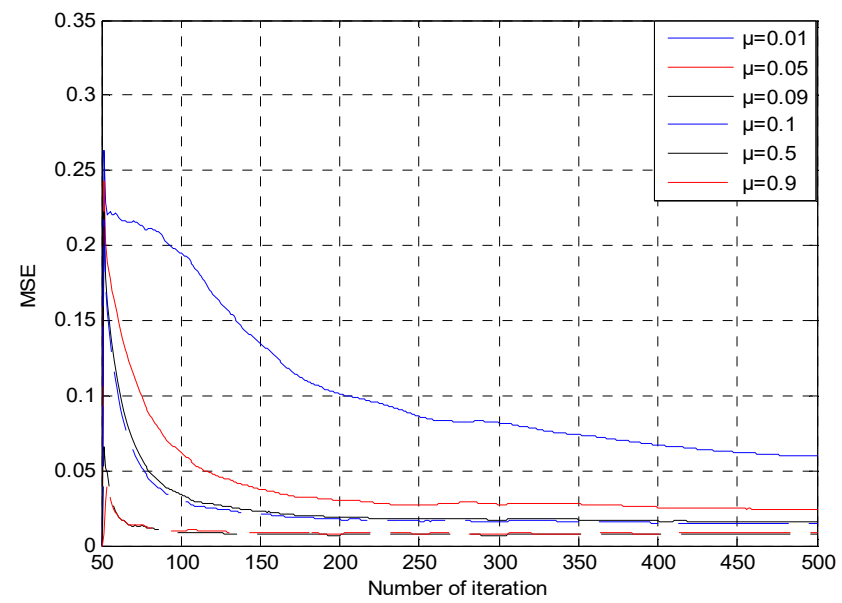

The performance of NLMS adaptive filter is analysed with different way to change the filter coefficients as mentioned in equation (1). The step size considered for evaluation is $0.01,0.05,0.09,0.1,0.5$ and 0.9 with fixed filter length. The performance of NLMS adaptive filter with given values of step size is depicted in Figure 8. The adaptation rate of NLMS algorithm is increasing as the step size is increasing. It requires less number of iteration to converge. The value of MSE is also reduced by increasing the value of step size. The step size 0.9 is required less number of iteration and performed better compared to other values of step size. The forecasting accuracy is also high at step size 0.9 as shown in Figure 9 with lower value of MAE. Therefore, for the next simulation setup the step size 0.9 is considered.

Figure 9 NLMS filter with different step size values (see online version for colours)

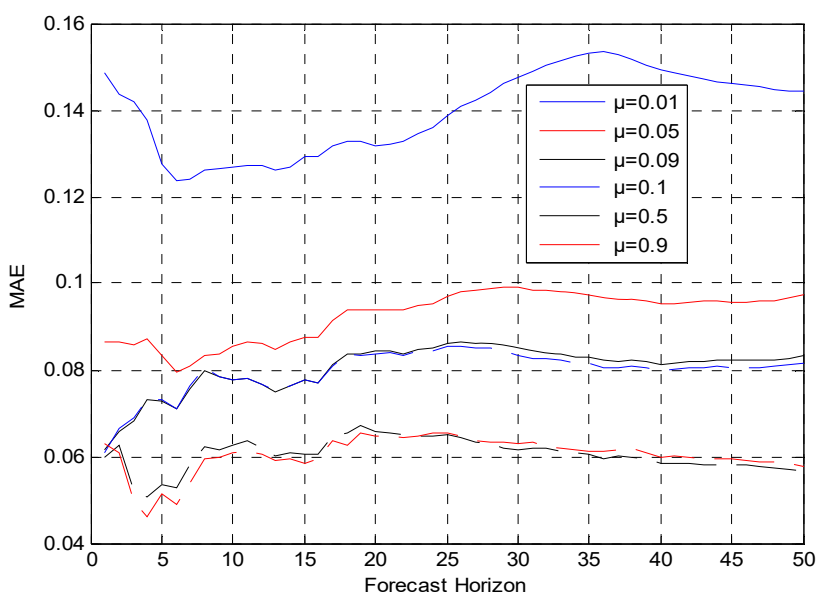


Figure 10 NLMS filter with different filter length values (see online version for colours)

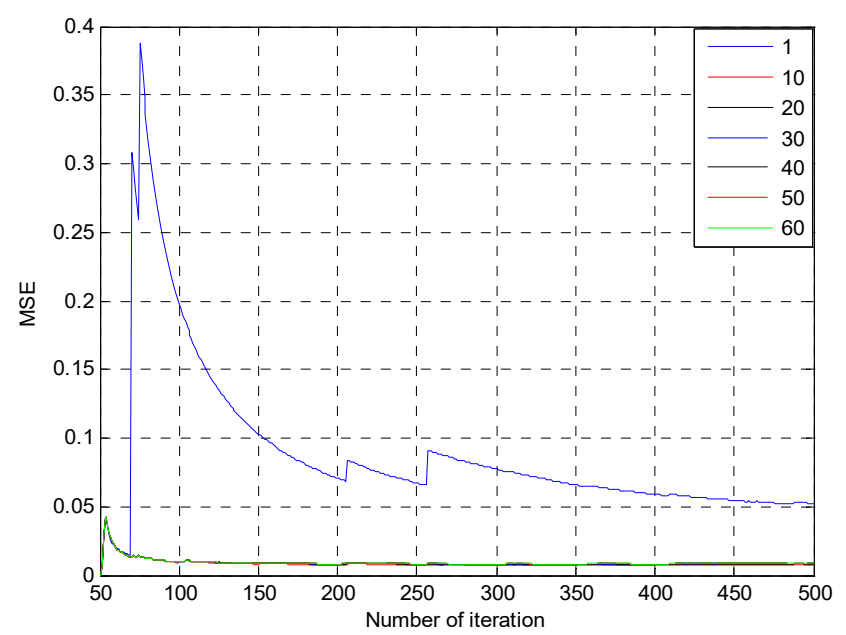

Figure 11 NLMS filter with different filter length values (see online version for colours)

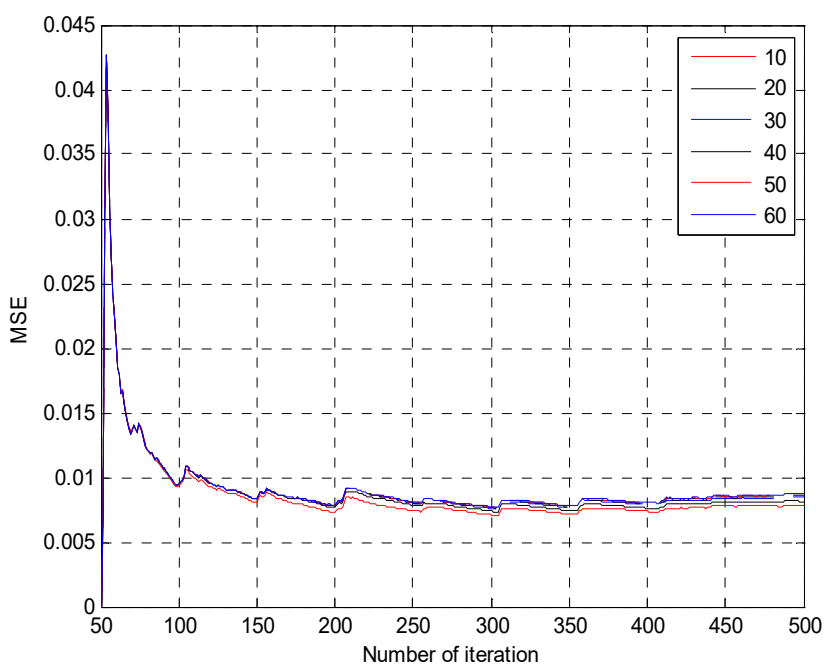

The NLMS adaptive filter is analysed with different filter length. The filter length is varying from 1 to 60 . The performance of NLMS filter with various filter length is shown in Figure 10. It is observed the difference in performance of NLMS algorithm with filter length 1 and 10, but after that increasing in the length of the filter, the difference in the value of MSE is not very large as observed by Figure 11. The change in filter length is also performed with prediction of IHR and is given in Figure 12. It is evaluated from the results that the prediction accuracy is high with filter length 20. Therefore in NLMS algorithm the step size 0.9 and filter length 20 performed better for ECG. 
Figure 12 NLMS filter with different filter length values (see online version for colours)

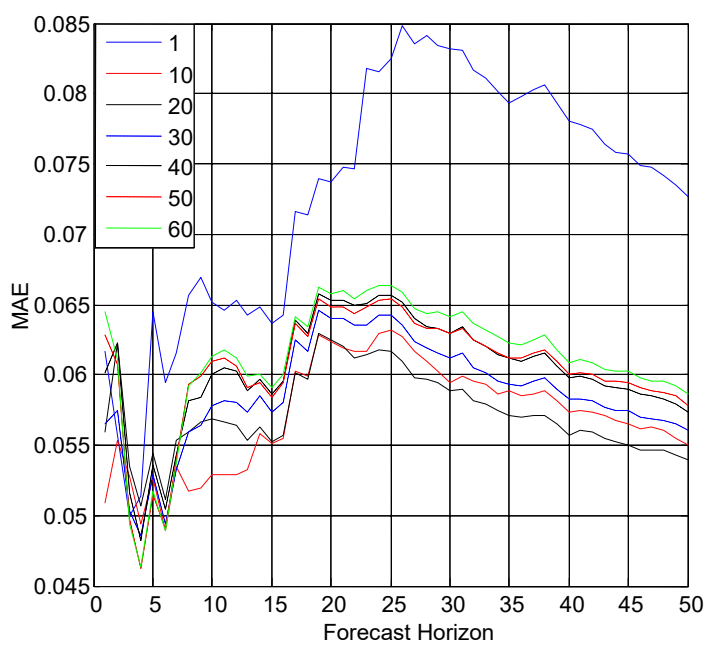

Figure 13 RLS filter with different forgetting factor values (see online version for colours)

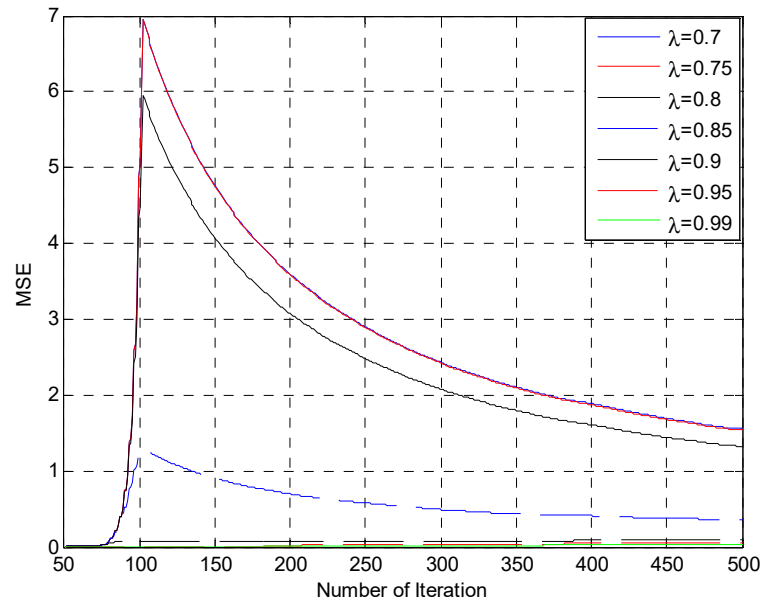

In case of RLS adaptive filter, the forgetting factor, which is related to past errors and filter length are responsible for the performance of prediction. The performance of RLS filter with different values of forgetting factor is shown in Figure 13 and Figure 14. The value of forgetting factor is $0.7,0.75,0.8,0.85,0.9,0.95$ and 0.99 considered. It is clear from the observation that as more number of past errors is considered in the RLS filter, the performance of the filter is better. Figure 15, shows the prediction of IHR by varying forgetting factor and it is also observed that at 0.99 the value of MAE in minimum. Therefore, with the value of forgetting factor 0.99 , the performance of RLS filter is evaluated by varying filter length. The assumed length of filter is from 1 to 60 as shown in =Figure 16. The variation in the performance and reduced the value of MAE, by increasing the length of RLS filter as depicted in Figure 17. The minimum value of MAE is achieved at filter length 60 . 
Figure 14 RLS filter with high values of forgetting factor (see online version for colours)

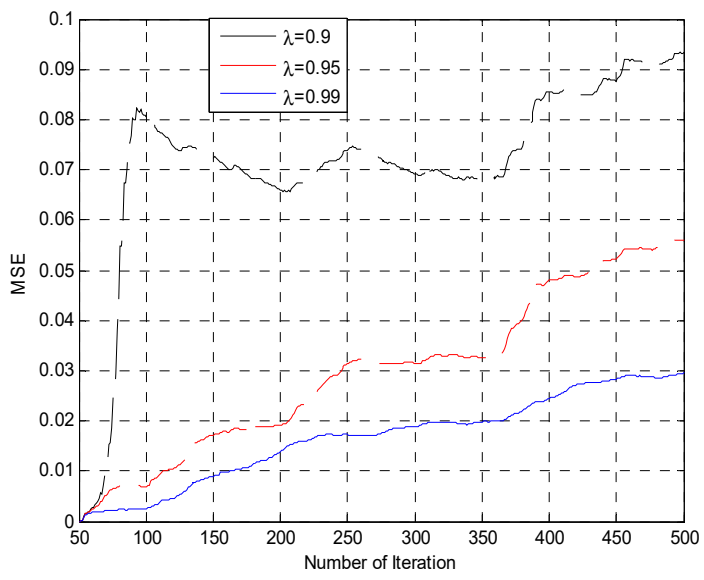

Figure 15 RLS filter with different forgetting factor values (see online version for colours)

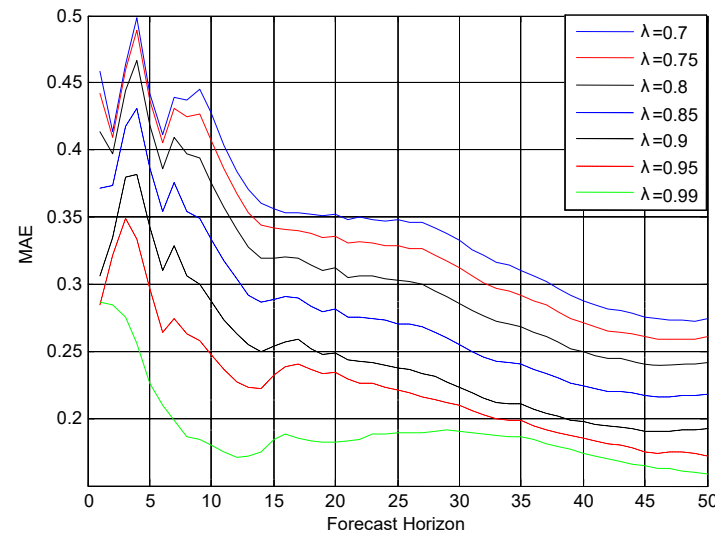

Figure 16 RLS filter with different filter length values (see online version for colours)

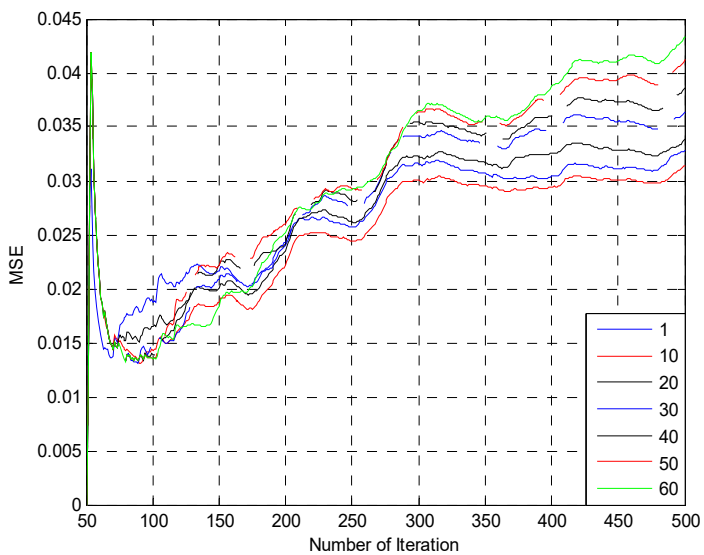


Figure 17 Prediction with different filter length values in RLS (see online version for colours)

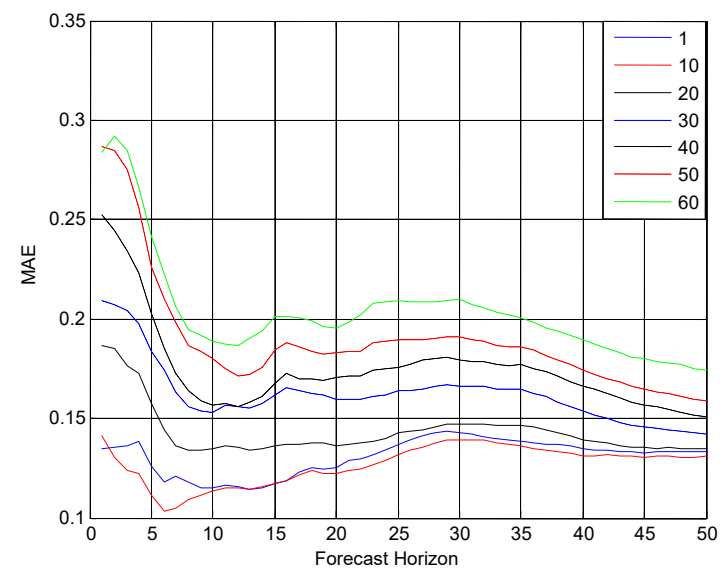

Figure 18 Comparison of adaptive filters (see online version for colours)

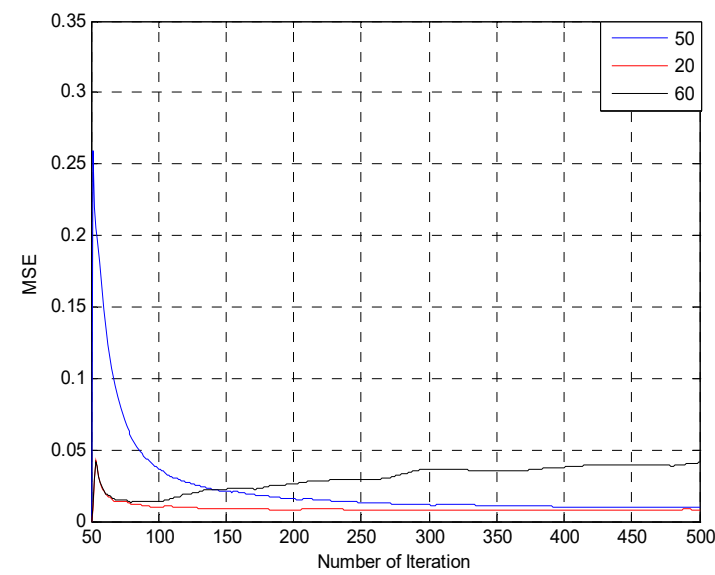

Figure 19 Prediction using adaptive filters (see online version for colours)

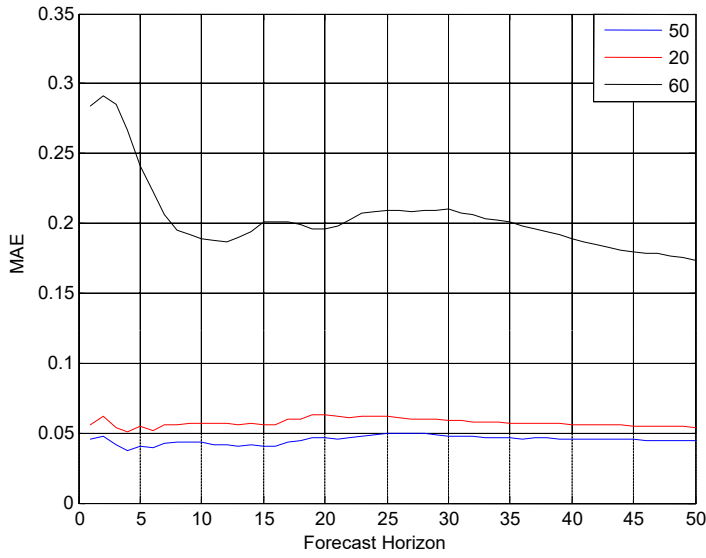


The step size of 0.05 for LMS algorithm, 0.9 for NLMS algorithm and the value of forgetting factor 0.99 is considered for RLS algorithm with filter length 50, 20, 60 respectively. The comparison between the adaptive filters using these algorithms with above mentioned step size, forgetting factor and filter length is shown in Figure 18. It is clear from Figure 18 that the convergence rate of NLMS and RLS algorithm is faster than the LMS algorithm. It means the NLMS and RLS algorithms are required less number of iteration to converge compared to LMS algorithm. The comparison is also done for prediction of IHR as depicted in Figure 19. It is evaluated that the LMS algorithm is performed better than the NLMS and RLS algorithm for prediction. Therefore, the prediction of IHR is more accurately performed by LMS adaptive filter compared to NLMS and RLS adaptive filters. When the discussion is related to the convergence rate and number of iteration required, the performance of RLS and NLMS adaptive filters are better compared to LMS adaptive filter but the high forecasting accuracy is achieved by LMS adaptive filter. The comparison of adaptive algorithms is presented in Table I, as per the observations and results. Table 2 contained the value of forecasting accuracy in terms of MAE for forecasting horizon 1 to 50. It is clear from the observation $\mathrm{s}$ that the error in terms of MAE is minimum in LMS for the prediction as compared to NLMS and RLS but the performance of NLMS and RLS algorithms in terms of convergence rate, number of iteration required etc. are better than LMS.

\section{Conclusions}

The novelty in this study is to apply the concept of time series analysis for prediction with adaptive algorithms for biomedical signal ECG. In this work adaptive filter was used to predict the ECG signal. The different markers of an ECG signal are playing an important role for diagnoses of many cardiovascular diseases and one of the markers is IHR, which was considered for prediction.

The adaptive filter is based on adaptive algorithms. The adaptive algorithms LMS, NLMS and RLS were used to predict value of IHR in ECG. The adaptive algorithms were analysed with various parameters of the filter like step size, number of iteration required for convergence, filter length etc. The results show that the high prediction accuracy is achieved by LMS algorithm with low value of MAE. The NLMS and RLS algorithms have faster convergence rate with less number of iteration. The results presented in this paper are the averaging of individual results of ECG record. The ECG records considered in this study belong to healthy persons.

\section{References}

Hayes, M.H. (1996) Statistical Digital Signal Processing and Modeling, John Wiley \& Sons Inc, New York, NY, USA.

Haykin, S. (2003) Adaptive Signal Processing, Wiley-Interscience, New York, NY, USA.

http://www.physionet.org/physiobank/database/nsrdb/

Kansal, S., Bansod, P.P. and Kumar, A. (2015) 'Statistical approach for determination of ECG markers', IEEE Conference on Computational Intelligence and Communication Networks (CICN).

Kwong, R. and Johnston, E.W. (1992) 'A variable step size LMS algorithm', IEEE Trans. On Signal Process., Vol. 40, No. 7, pp.1633-1642. 
Liang, F., Yu, Y., Wang, H. and Meng, X. (2013) 'Heart motion prediction in robotic assisted beating heart surgery: a nonlinear fast adaptive approach', Int. J. Adv. Robotic Sys., Vol. 10, Article No. 129.

Moschytz, G. and Hofbauer, M. (2000) Adaptive Filter, Springer Verlag, Berlin, ISBN 3-54067651-1.

Naga Rajesh, A., Chandralingam, S., Anjaneyulu, T. and Satyanarayana, K. (2012) 'Performance evaluation of adaptive filter algorithms for EOG signal processing', International Journal of Systems, Algorithms \& Applications, Vol. 2, pp.14-18, ISSN No. 2277-2677.

Shams, M., Abadi, E. and Moradiani, F. (2012) 'A unified approach to tracking performance analysis of the selective partial update adaptive filter algorithms in nonstationary environment', Digital Signal Processing, Elsevier.

Song, J., Dan, L., Xiaoyuan, M., Teng, G. and Wei, J. (2017) 'A robust dynamic heart-rate detection algorithm framework during intense physical activities using photoplethysmographic signals', Published in Sensors, Vol. 17, No. 11, pp.1-17.

Stojkoska, B., Solev, D. and Davcev, D. (2011) 'Data prediction in WSN using variable step size LMS algorithm', The Fifth International Conference on Sensor Technologies and Applications, ENSORCOMM, pp.191-196.

Tuna, E.E. et al. (2013) 'Heart motion prediction based on adaptive estimation algorithms for robotic assisted beating heart surgery', IEEE Trans. on Robotics, Vol. 29, No. 1, pp.261-276.

Wesen, J.E. (2015) Adaptive Filter Design for Stock Market Prediction Using a Correlation-Based Criterion [online] https://arxiv.org/abs/1501.07504.

Widrow, B. and Stearns, S.D. (1985) Adaptive Signal Processing, Prentice Hall, Upper Saddle River, NJ.

Widrow, S.B. and Hoff, M.E. (1960) 'Adaptive switching circuits', IRE WESCON Conv. Rec., Vol. 4, pp.96-104.

Windrow, B. and Stearns, S.D. (2007) Adaptive Signal Processing, Pearson Education, Inc, NJ, USA.

Zia, M.G. (2009) 'Channel estimation using adaptive filtering in OFDM system', Proceedings of the IASTED International Conference Signal and Image Processing, pp.25-27. 